\title{
Separate Neural Substrates in the Human Cerebellum for Sensory-motor Adaptation of Reactive and of Scanning Voluntary Saccades
}

N. Alahyane, V. Fonteille, C. Urquizar, : Salemme, N. Nighoghossian, Denis Pelisson, C. Tilikete

\section{To cite this version:}

N. Alahyane, V. Fonteille, C. Urquizar, : Salemme, N. Nighoghossian, et al.. Separate Neural Substrates in the Human Cerebellum for Sensory-motor Adaptation of Reactive and of Scanning Voluntary Saccades. The Cerebellum, Taylor

Francis Ltd., 2008, 7 (4), pp.595-601. 10.1007/s12311-008-0065-5 . hal-02197091

\section{HAL Id: hal-02197091 \\ https://hal.archives-ouvertes.fr/hal-02197091}

Submitted on 30 Jul 2019

HAL is a multi-disciplinary open access archive for the deposit and dissemination of scientific research documents, whether they are published or not. The documents may come from teaching and research institutions in France or abroad, or from public or private research centers.
L'archive ouverte pluridisciplinaire HAL, est destinée au dépôt et à la diffusion de documents scientifiques de niveau recherche, publiés ou non, émanant des établissements d'enseignement et de recherche français ou étrangers, des laboratoires publics ou privés. 


\title{
Separate Neural Substrates in the Human Cerebellum for Sensory-motor Adaptation of Reactive and of Scanning Voluntary Saccades
}

\author{
N. Alahyane - V. Fonteille - C. Urquizar • R. Salemme • \\ N. Nighoghossian • D. Pelisson • C. Tilikete
}

\begin{abstract}
Sensory-motor adaptation processes are critically involved in maintaining accurate motor behavior throughout life. Yet their underlying neural substrates and taskdependency bases are still poorly understood. We address these issues here by studying adaptation of saccadic eye movements, a well-established model of sensory-motor plasticity. The cerebellum plays a major role in saccadic adaptation but it has not yet been investigated whether this role can account for the known specificity of adaptation to
\end{abstract}

N. Alahyane $\cdot$ V. Fonteille $\cdot$ C. Urquizar $\cdot$ R. Salemme $\cdot$

D. Pelisson $\cdot$ C. Tilikete

INSERM, U864, Espace et Action,

Bron 69500, France

N. Alahyane $\cdot$ V. Fonteille $\cdot$ C. Urquizar $\cdot$ R. Salemme $\cdot$

N. Nighoghossian $\cdot$ D. Pelisson $\cdot$ C. Tilikete

Université de Lyon,

Lyon 69003, France

N. Alahyane $\cdot$ V. Fonteille $\cdot C$. Urquizar $\cdot$ R. Salemme $\cdot$

N. Nighoghossian $\cdot$ D. Pelisson $\cdot$ C. Tilikete

Université Lyon 1, Biologie Humaine,

Lyon 69003, France

N. Alahyane $\cdot$ V. Fonteille $\cdot$ C. Urquizar $\cdot$ R. Salemme $\cdot$

D. Pelisson $\cdot$ C. Tilikete

Hospices Civils de Lyon, Mouvement et Handicap,

Hôpital Neurologique,

Lyon 69003, France

N. Alahyane $\cdot$ V. Fonteille $\cdot$ C. Urquizar $\cdot$ R. Salemme $\cdot$

D. Pelisson $\cdot$ C. Tilikete

IFR19, Institut Fédératif des Neurosciences de Lyon,

Lyon 69003, France

N. Alahyane $\cdot$ V. Fonteille $\cdot$ C. Urquizar $\cdot$ R. Salemme $\cdot$ D. Pelisson $\cdot$ C. Tilikete

IFR23, Institut Fédératif de Recherche sur le Handicap, Bron 69500, France the saccade type (e.g., reactive versus voluntary). Two patients with focal lesions in different parts of the cerebellum were tested using the double-step target paradigm. Each patient was submitted to two separate sessions: one for reactive saccades (RS) triggered by the sudden appearance of a visual target and the second for scanning voluntary saccades (SVS) performed when exploring a more complex scene. We found that a medial cerebellar lesion impaired adaptation of reactive - but not of voluntary — saccades,

N. Nighoghossian

Hospices Civils de Lyon, Cerebrovascular Unit,

Hôpital Neurologique,

Lyon, France

N. Nighoghossian

Creatis UMR CNRS 5515 INSERM U630, UCB Lyon I,

Lyon, France

D. Pelisson $(\bowtie)$

"Espace et Action" U864, INSERM, University Lyon 1,

16 avenue du doyen Lépine,

69676 Bron cedex, France

e-mail: pelisson@lyon.inserm.fr

Present address:

N. Alahyane

Centre for Neuroscience Studies, Queen's University,

Botterell Hall, Room 234,

Kingston, ON, Canada K7L 3N6

Present address:

V. Fonteille

Inserm, U742, Université Pierre et Marie Curie,

Paris 75005, France 
whereas a lateral lesion affected adaptation of scanning voluntary saccades, but not of reactive saccades. These findings provide the first evidence of an involvement of the lateral cerebellum in saccadic adaptation, and extend the demonstrated role of the cerebellum in RS adaptation to adaptation of SVS. The double dissociation of adaptive abilities is also consistent with our previous hypothesis of the involvement in saccadic adaptation of partially separated cerebellar areas specific to the reactive or voluntary task (Alahyane et al. Brain Res 1135:107-121 (2007)).

Keywords Sensory-motor - Adaptation · Cerebellum · Saccades $\cdot$ Plasticity $\cdot$ Neuropathology

\section{Introduction}

The cerebellum is known as the main neural center for cellular plasticity underlying long-term adaptation of motor behavior, but its precise role is still intensively debated [25]. In particular, whether the task specificity of sensorymotor adaptation [5-9] relies on the selective involvement of different adaptable cerebellar circuits is still unknown. We addressed this issue using saccadic adaptation-a progressive adjustment of the amplitude of saccadic eye movements to a systematic perturbation of target positionas a key model of sensory-motor adaptation [8]. Previous works suggested that adaptation of reactive saccades (RS) elicited by the sudden presentation of a novel visual target critically depends on the medial part of the posterior (MP) cerebellum [10-13]. In contrast, adaptation of scanning voluntary saccades (SVS) has been much less studied, although these endogenously generated saccades are predominant in daily life. Based on the pattern of adaptation transfer between RS and SVS in healthy humans, we recently suggested that SVS and RS adaptations rely both on a common brainstem site and on two separate, but partly overlapping, cerebellar sites [1]. We provide here direct evidence for this hypothesis by reporting on saccadic adaptation abilities in two volunteers with isolated cerebellar lesion (Fig. 1a). Indeed, this case study disclosed two opposite patterns of adaptation deficits for reactive and voluntary saccades depending on the lesioned cerebellar site.

\section{Materials and Methods}

\section{Patients}

P1, a 40-year-old man, was tested 10 days after a stroke located at the intermediate level of the rostral right hemisphere of the cerebellum (infarct of antero-inferior cerebellar artery). P2, a 61-year-old man, was tested
4 months after a stroke affecting the caudal paramedian part of the right cerebellar hemisphere (infarct of the medial branch of the postero-inferior cerebellar artery). Note that the available imaging data did not allow us to delineate very clearly the lesions' boundaries. However, it is obvious that the two lesions were clearly separate as they involved the lateral part of anterior lobe in P1 and the medial part of posterior lobe in $\mathrm{P} 2$. At the time of recording, a right-sided limb ataxia (P1) or axial ataxia (P2) were still present, but there was no ocular nystagmus.

\section{Experimental Set-up}

The set-up and protocols were approved by the local ethics committee (CCPPRB-Lyon B) and have been described in detail in a previous paper [1]. Visual targets $(6 \mathrm{~mm}$ black dots on a gray background) were presented on a video screen by means of a 142-Hz visual stimulus generation system (CRS, Cambridge, UK). A helmet-mounted EyeLink II eye-tracker (SR Research, Mississauga, Canada) was used to record 2-D binocular eye movements (500 Hz sampling, accuracy better than $0.1^{\circ}$ ). Real-time monitoring of eye position allowed us to modify the visual display during primary saccades within $10 \mathrm{~ms}$ of a pre-defined event trigger (i.e., eye velocity exceeding a $100 \%$ s threshold).

\section{Procedure}

After providing written informed consent, each patient underwent RS and SVS adaptations in separate sessions. A single target (RS) or a set of targets (SVS) shifted backward during each primary saccade (Fig. 1b,c), in order to induce an adaptive gain reduction of both leftward and rightward saccades. As a consequence of saccadic suppression [14, 15], such target shifts were unnoticed by the patients. Adaptation after-effect was measured during blocks of unperturbed trials, i.e., without intra-saccadic target shift, before ('pre') and after ('post') completing five blocks of the adaptation run ('ada1' to 'ada5').

$R S$ session The adaptation of RS was tested in this session. The first adaptation blocks comprised 48 trials each (24 leftward saccades randomly intermingled with 24 rightward saccades). Each adaptation trial (Fig. 1b) started with the presentation of a central fixation point (FP) for a pseudorandom period $(1,300$ or 1,500 or $1,700 \mathrm{~ms})$. Then, FP was replaced by a target (T1) located randomly at $8^{\circ}$ to the right or to the left $\left( \pm 8^{\circ}\right)$. During the ensuing horizontal RS, T1 shifted back to $\mathrm{T} 1^{\prime}$ by $25 \%$ of the initial target displacement for blocks ada1, ada2, and ada3 (144 trials), and by $40 \%$ for blocks ada4 and ada5 (96 trials). Finally, T1' was switched off $800 \mathrm{~ms}$ after T1 onset, and FP re-appeared 1,200 ms later for the next trial. Pre-adaptation and post-adaptation 
Fig. 1. MRI scans and adaptation protocols. a FLAIR (patient P1) and diffusion (patient P2) images showing stroke lesions (arrows) located at the intermediate level of the rostral right hemisphere (P1) and at the caudal paramedian part of the right hemisphere (P2). b RS adaptation for a rightward trial. Peripheral target $\mathrm{T} 1$ turned on simultaneously when fixation point FP turned off (rightward arrow), eliciting a primary saccade. During this saccade, T1 shifted backward to T1' (leftward arrow). c SVS adaptation for a rightward trial. Subjects sequentially scanned targets (from T0 to T3) at their own pace. During each of the three SVS, the whole display shifted backward (leftward arrows) until reaching the $\mathrm{T}^{\prime \prime \prime}-\mathrm{T} 3^{\prime \prime \prime}$ positions

\section{a}

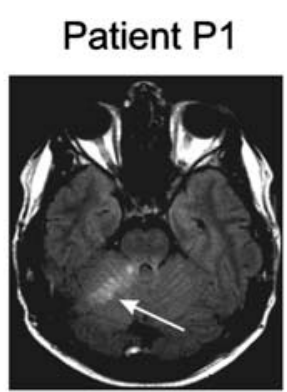

Right

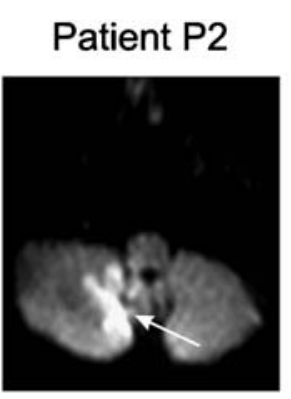

Right

Left

b

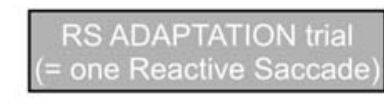

Initial target step

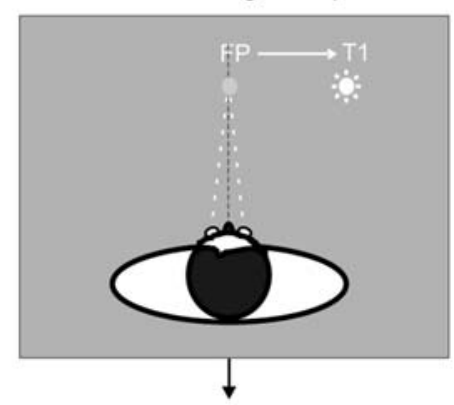

Intra-saccadic target shift

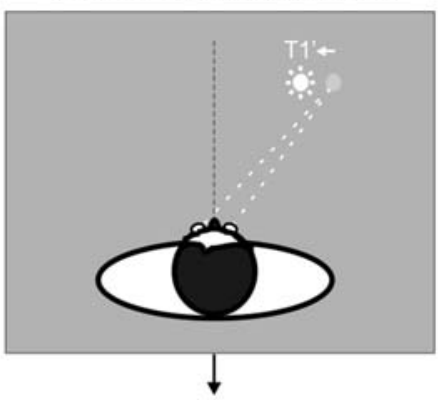

Fixation of shifted target

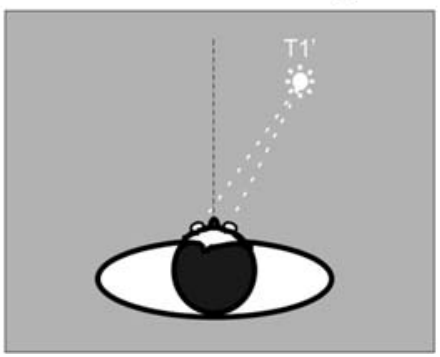

C

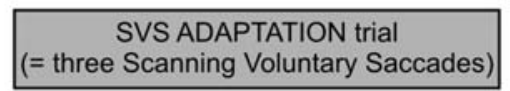

Initial target display

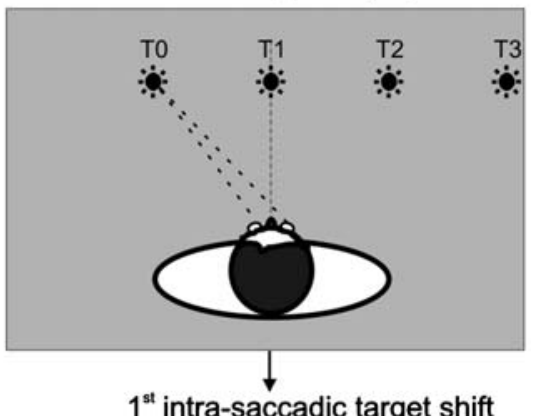

$1^{\text {st }}$ intra-saccadic target shift

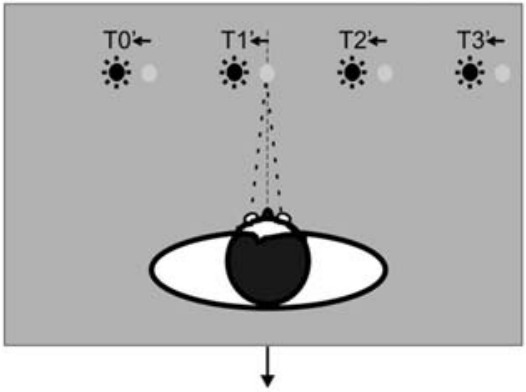

$1^{\text {st }}$ fixation of shifted targets (T1')

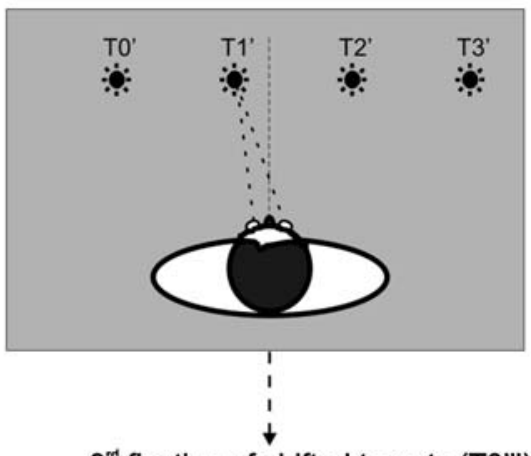

$3^{\text {rd }}$ fixation of shifted targets (T3"')

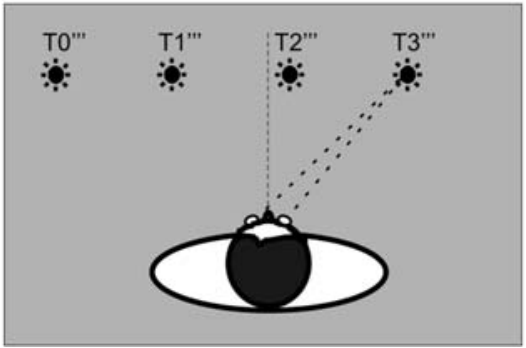

trials were identical to adaptation trials, except that target T1 did not shift but instead disappeared during primary saccades. Each test block comprised 12 leftward saccades randomly intermingled with 12 rightward saccades.

SVS session The adaptation of SVS was tested in this second session. The adaptation blocks comprised 16 trials of three SVS each. Each adaptation trial (Fig. 1c) started with the presentation of FP at $8^{\circ}$ to the right (leftward trial) or left (rightward trial) of the screen center and $4^{\circ}$ above. After 1,600 ms, a circle surrounding FP was presented simultaneously with targets T0 to T3 (T0 located below FP) along the central horizontal axis with an $8^{\circ}$ regular spacing. The circle disappeared $500 \mathrm{~ms}$ later, signalling subjects to 
perform a downward saccade to T0 followed by a sequence of three horizontal SVS. During each of these SVS, the entire set of targets was shifted horizontally backward by $25 \%$ of the horizontal target separation for blocks ada1, ada 2 , and ada 3 and by $40 \%$ for blocks ada 4 and ada5. Preadaptation and post-adaptation trials differed from adaptation trials in two aspects. First, only three targets (FP, T0, and T1) were presented: T0 at the center of the screen, FP at $4^{\circ}$ above $\mathrm{T} 0$, and $\mathrm{T} 1$ at $-8^{\circ}$ (left) or $+8^{\circ}$ (right) from T0. Subjects thus performed a vertical saccade from FP to T0, followed by a single horizontal SVS from T0 to T1. Second, this display did not shift but instead disappeared during the SVS. Each 'pre' and 'post' block was subdivided in two sub-blocks of 12 rightward and 12 leftward trials.

\section{Data Analysis}

Signals from the two eyes were averaged to yield the cyclopean eye position. After automatic detection and visual check of all primary saccades, the horizontal saccade gain (=ratio of eye displacement amplitude to target eccentricity) was submitted to one-way ANOVAs with the "trial block" factor (seven levels = 'pre', 'ada1'-'ada5', and 'post') separately for P1 and P2, for RS and SVS, and for rightward and leftward saccades. Post hoc tests (LSD Fisher) compared saccade gain during 'ada' blocks and 'post' blocks to the 'pre' block. Finally, the adaptation after-effect [100*('pre' gain- 'post' gain)/ 'pre' gain] was compared to the $95 \%$ confidence intervals computed in six healthy subjects from our previous study [1].

\section{Results}

Table 1 summarizes the mean baseline gain of RS and SVS measured during the pre-adaptation block. For statistical analysis, these data were compared to control data collected under the same conditions in a group of six healthy subjects (age range 22-48 years, two women, four men; see [1]). Saccade gains in patients were considered as abnormal (see arrows in the table) if the means were outside the $95 \%$ confidence intervals computed from the control mean gains. These comparisons revealed that saccades in patients were either normometric or hypometric, with the exception of a very moderate gain increase for contralesional RS in P1. Note that neither this pattern of dysmetria nor the betweensubjects variations of gain variability (standard deviation, s.d.) closely paralleled the pattern of adaptation deficits presented in the following text.

\section{Time-course of Saccadic Gain Changes}

Figure $2 \mathrm{a}$ shows the changes in saccade gain in the five adaptation blocks and the post-adaptation block relative to pre-adaptation. Each patient showed a specific pattern of adaptation depending on the direction (ipsilesional versus contralesional) and type (RS versus SVS) of saccade. For the contralesional direction, both patients showed a decrease in RS and SVS gain in both adaptation and postadaptation blocks, consistent with the expected effect of backward adaptation training. ANOVAs confirmed a significant effect of the "trial block" factor ( $F=8$ to $41, P<0.001$; post hoc comparisons with the 'pre' block shown in Fig. 2a). These gain reductions followed a time course closely resembling that of healthy subjects [1] and revealed that adaptation of contralesional saccades was preserved in P1 and P2. For ipsilesional saccades, a different picture emerged. P1 exhibited preserved adaptation abilities for RS, as shown by the significant decrease of gain in adaptation and post-adaptation blocks (ANOVA, $F=23, P<0.001$ ). However, P1's adaptation abilities for SVS were altered: the gain change during adaptation $(F=7, P<0.001)$ was moderate and inconsistent between blocks, and it was not maintained in post-adaptation (post hoc comparison with 'pre', $P>0.05)$. P2 showed the opposite pattern: SVS adaptation was fully preserved $(F=10, P<0.001)$, whereas RS adaptation was strongly impaired both during the adaptation and post-adaptation phases $(F=2, P>0.15)$.

\section{Adaptation After-effect}

We then compared the adaptation after-effect in P1 and P2 (Fig. 2b) to 95\% confidence intervals computed from our previous study in six healthy subjects [1]. For the contralesional direction, both RS and SVS after-effects were within control confidence intervals, strengthening our conclusion that saccadic adaptation was normal despite the cerebellar lesions. In contrast, for the ipsilesional direction, adaptation after-effects were significantly smaller for SVS in P1, and for

Table 1 Mean (s.d.) baseline saccade gain measured in the pre-adaptation block

\begin{tabular}{|c|c|c|c|c|}
\hline & Ipsilesional RS & Ipsilesional SVS & Contralesional RS & Contralesional SVS \\
\hline Patient P1 & $0.89(0.11)$ & $0.83(0.06)$ ת & $0.98(0.06)$ 仓 & $0.94(0.12)$ \\
\hline Patient P2 & $0.76(0.13)$ & $0.92(0.03)$ & $0.76(0.09)$ ת ת & $0.91(0.03)$ \\
\hline
\end{tabular}

ת and $\hat{\imath}$ : statistically significant decrease or increase $(P<0.05)$ relative to controls' $95 \%$ confidence intervals. see Ref. [1] 


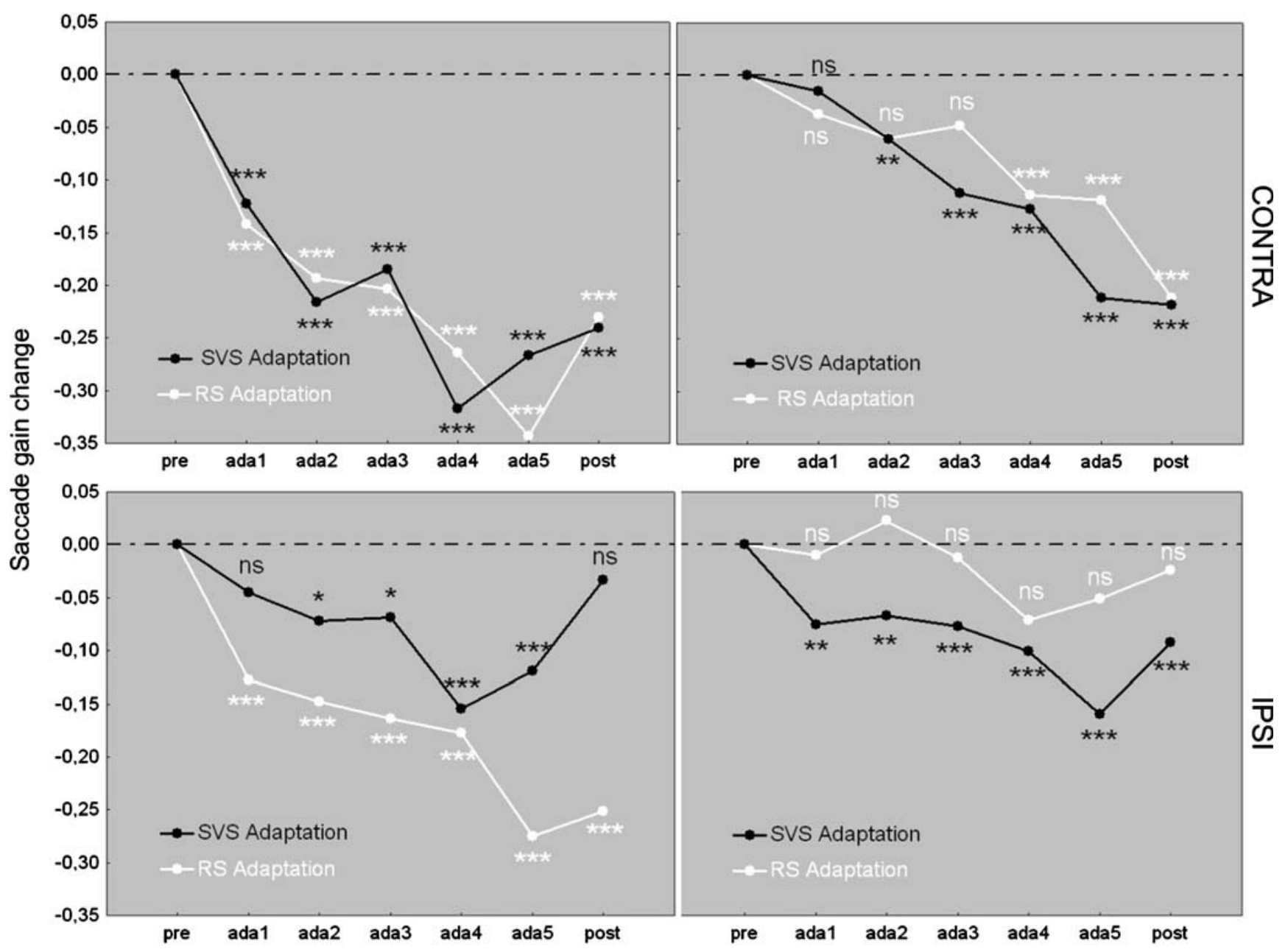

Blocks of trials

b
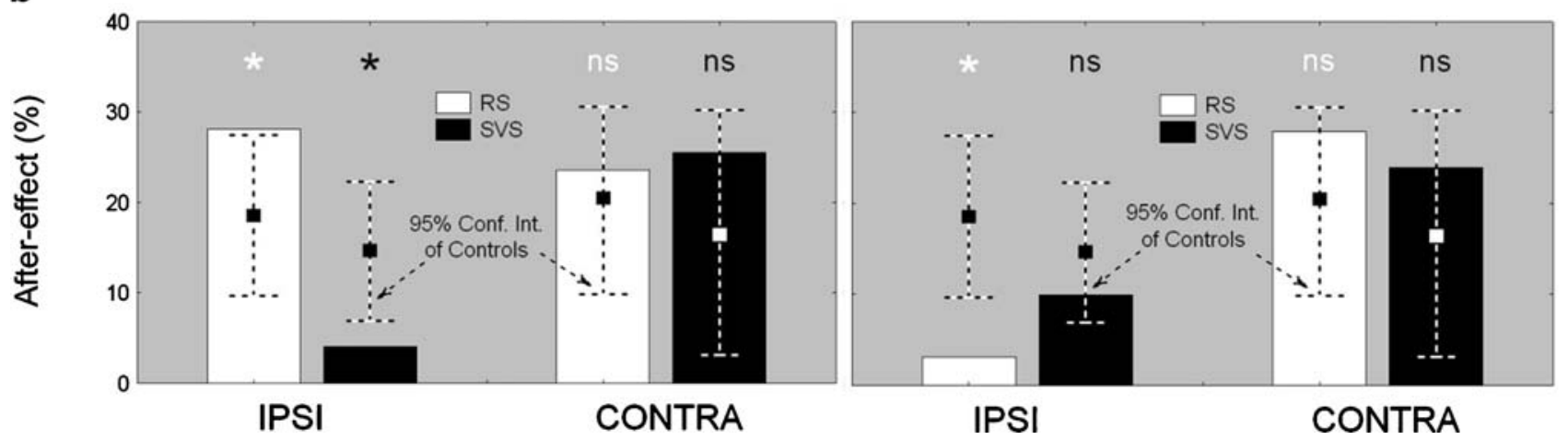

Fig. 2. Adaptation abilities of patients $P 1$ and $P 2$. a Mean gain change of contralesional (top) and ipsilesional (bottom) saccades in adaptation ('ada1-ada5') and post-adaptation ('post') trial blocks relative to the 'pre' block. Negative values indicate a gain decrease whereas positive values indicate a gain increase. $* P<0.05, * * P<0.01$, $* * * P<0.001$, ns $P>0.05$ (post hoc LSD Fisher tests comparing saccade gains to the 'pre' saccade gain). b Adaptation after-effect: mean percent gain change $(100 *$ ('pre'-'post')/ pre') for RS (white bar) and SVS (black bar) computed in patients. These values were statistically compared to the means (filled squares) and $95 \%$ confidence intervals (whiskers) computed in six healthy subjects from our previous study [1]: ${ }^{*} P<0.05$, ns $P>0.05$ 
RS in P2 (and marginally larger for RS in P1) as compared to controls. Thus, the reduced levels of ipsilesional saccade adaptation described above (Fig. 2a) did not result from idiosyncratic fluctuations but rather from the cerebellar lesion itself.

\section{Discussion}

The double dissociation observed for ipsilesional saccades reveals that medial (patient P2) and lateral (patient P1) parts of the cerebellum selectively participate in RS and in SVS adaptation, respectively. These findings confirm the contribution of the MP cerebellum to RS adaptation [10-13]. More importantly, they demonstrate for the first time that cerebellar areas located more laterally than the MP cerebellum also contribute to saccadic adaptation (SVS). Remarkably, a role of the lateral cerebellum in saccade control has so far remained elusive [16], notably because the effects of lesions to this area are weak or absent. Thus, the function of both saccade-related activity and oculomotor outputs of these lateral areas are still a mystery. The present study reveals that one possible function is the adaptive control of SVS, in line with the recent proposal of a lateral cerebellum specialization for voluntary saccades [17]. Unfortunately, for practical reasons, RS and SVS adaptation could only be tested once in each patient, preventing us from testing for the consistency of the present findings. However, despite the known variability of saccadic adaptation from session to session, the deficits reported in the patients do likely result specifically from their lesion because contralesional saccade gain always adapted normally.

Our second original finding is that RS and SVS adaptations rely upon separate neural substrates in the cerebellum, which directly supports our predictions [1]. Recent studies in healthy human subjects have measured the pattern of adaptation transfer either between RS and SVS [1, 18] or from RS and SVS to hand pointing movements [19] and to antisaccades (Cotti et al., submitted) and have all proposed that RS and SVS adaptation rely on separate mechanisms. The present study confirms this proposal by revealing, in the human cerebellum, a neural substrate of this functional separation between the two types of saccadic adaptation. Further recordings in groups of patients must be performed to more clearly define the lateral and medial cerebellar areas involved respectively in SVS and RS adaptation. High resolution 3-D imaging of the cerebellum would thus be necessary to establish detailed anatomo-functional correlations. Note also that the contribution of these lateral and medial cerebellar areas may not be the same with respect to the acquisition and/or retention of adaptation, as suggested by the present observations. In any case, with this unique anatomofunctional organization, the cerebellum may not only implement plasticity related to adaptation per se, but may also process task-related signals to switch between different adapted states, as previously suggested for limb motor responses in animals $[20,21]$. This advance in knowledge of the selective and plastic functions housed in the cerebellum will help our understanding of how the central nervous system maintains, over the long term, proficient visuo-motor interactions with our environment.

Acknowledgements Work performed in the "Mouvement et Handicap" IFNL platform (Lyon, France). Supported by "Hospices Civils de Lyon" (HCL/P 2002.303), INSERM U864, CNRS (DP), and a FRM fellowship (NA). Thanks to A. Farnè, N. Holmes, and B. White for valuable comments.

\section{References}

1. Alahyane N, Salemme R, Urquizar C, Cotti J, Guillaume A, Vercher J-L, Pélisson D (2007) Oculomotor plasticity: are mechanisms of adaptation for reactive and voluntary saccades separate. Brain Res 1135:107-121

2. Ito M (1984) The cerebellum and neural control. Raven, New York

3. Ito M (2006) Cerebellar circuitry as a neuronal machine. Prog Neurobiol 78:272-303

4. Schmahmann JD (1997) The cerebellum and cognition. Academic, San Diego

5. Bloedel JR (2004) Task-dependent role of the cerebellum in motor learning. Prog Brain Res 143:319-329

6. Deubel H (1995) Separate adaptive mechanisms for the control of reactive and volitional saccadic eye movements. Vis Res 35: $3529-3540$

7. Krakauer JW, Ghilardi MF, Ghez C (1999) Independent learning of internal models for kinematic and dynamic control of reaching. Nat Neurosci 2:1026-1031

8. Hopp JJ, Fuchs AF (2004) The characteristics and neuronal substrate of saccadic eye movement plasticity. Prog Neurobiol 72:27-53

9. Wainscott SK, Donchin O, Shadmehr R (2005) Internal models and contextual cues: encoding serial order and direction of movement. J Neurophysiol 93:786-800

10. Desmurget M, Pélisson D, Urquizar C, Prablanc C, Alexander GE, Grafton ST (1998) Functional anatomy of saccadic adaptation in humans. Nat Neurosci 1:524-528

11. Takagi M, Zee DS, Tamargo RJ (1998) Effects of lesions of the oculomotor vermis on eye movements in primate: saccades. J Neurophysiol 80:1911-1931

12. Barash S, Melikyan A, Sivakov A, Zhang M, Glickstein M, Thier P (1999) Saccadic dysmetria and adaptation after lesions of the cerebellar cortex. J Neurosci 19:10931-10939

13. Straube A, Deubel H, Ditterich J, Eggert T (2001) Cerebellar lesions impair rapid saccade amplitude adaptation. Neurology 57:21052108

14. Volkmann FC, Schick AM, Riggs LA (1968) Time course of visual inhibition during voluntary saccades. J Opt Soc Am 58:562-569

15. Goodale MA, Pélisson D, Prablanc C (1986) Large adjustments in visually guided reaching do not depend on vision of the hand or perception of target displacement. Nature 320:748-750

16. Pélisson D, Goffart L, Guillaume A (2003) Control of saccadic eye movements and combined eye/head gaze shifts by the medioposterior cerebellum. Prog Brain Res 42:69-89 
17. Simo LS, Krisky CM, Sweeney JA (2005) Functional neuroanatomy of anticipatory behavior: dissociation between sensorydriven and memory-driven systems. Cereb Cortex 15:19821991

18. Collins T, Dore-Mazars K (2006) Eye movement signals influence perception: evidence from the adaptation of reactive and volitional saccades. Vis Res 46:3659-3673

19. Cotti J, Guillaume A, Alahyane N, Pélisson D, Vercher JL (2007) Adaptation of voluntary saccades, but not of reactive saccades, transfers to hand pointing movements. J. Neurophysiol 98:60212

20. Lewis RF (2003) Context-dependent adaptation of visually-guided arm movements and vestibular eye movements: role of the cerebellum. Cerebellum 2:123-130

21. Shimansky Y, Wang JJ, Bauer RA, Bracha V, Bloedel JR (2004) On-line compensation for perturbations of a reaching movement is cerebellar dependent: support for the task dependency hypothesis. Exp Brain Res 155:156-172 